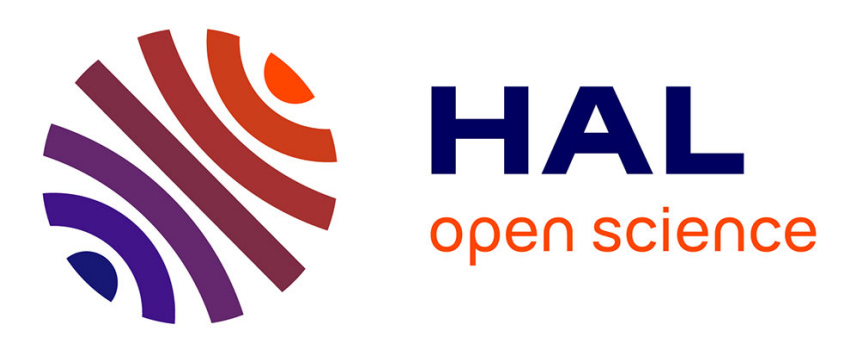

\title{
On the fixed controllable subspace in linear structured systems
}

Christian Commault, Jacob van Der Woude, Taha Boukhobza

\section{To cite this version:}

Christian Commault, Jacob van Der Woude, Taha Boukhobza. On the fixed controllable subspace in linear structured systems. Systems and Control Letters, 2017, 102, pp.42-47. 10.1016/j.sysconle.2017.01.002 . hal-01480697

\section{HAL Id: hal-01480697 https://hal.science/hal-01480697}

Submitted on 7 Mar 2017

HAL is a multi-disciplinary open access archive for the deposit and dissemination of scientific research documents, whether they are published or not. The documents may come from teaching and research institutions in France or abroad, or from public or private research centers.
L'archive ouverte pluridisciplinaire HAL, est destinée au dépôt et à la diffusion de documents scientifiques de niveau recherche, publiés ou non, émanant des établissements d'enseignement et de recherche français ou étrangers, des laboratoires publics ou privés. 


\title{
On the fixed controllable subspace in linear structured systems
}

\author{
Christian Commault ${ }^{\mathrm{a}}$, Jacob van der Woude $^{\mathrm{b}}$, Taha Boukhobza ${ }^{\mathrm{c}}$ \\ ${ }^{a}$ Univ. Grenoble Alpes, CNRS, GIPSA-lab, F-38000 Grenoble, France. \\ Email: christian.commault@grenoble-inp.fr \\ ${ }^{b}$ DIAM, EWI, Delft University of Technology, Mekelweg 4, 2628 CD, Delf \\ the Netherlands. \\ Email: j.w.vanderwoude@tudelft.nl \\ ${ }^{c}$ Université de Lorraine, CRAN, UMR 7039, France \\ CNRS, CRAN, UMR 7039, France \\ BP 70239 - 54506 VANDOEUVRE Cedex. \\ Email:taha.boukhobza@univ-lorraine.fr
}

\begin{abstract}
In this paper we consider interconnected networks that are described by means of structured linear systems with state and control variables. We represent these systems, whose matrices contain fixed zeros and free parameters, by means of directed graphs and study questions concerning controllability and the controllable subspace.

We show in this paper that the controllable subspace can have a part that will be present for almost all values of the free parameters. It actually is a subspace of the controllable subspace and will be referred to as the fixed controllable subspace. The subspace can then be seen as a kind of robustly controllable part of the system. Indeed, it is a subspace in the state space with the generic property that states in it can be steered in an arbitrary way.

We derive a characterization of the fixed controllable subspace using the graph representation. The obtained characterization makes use of well-known algorithms from optimization and networks theory. To get some more insight in the components in the fixed part, we also give a representation of the structured linear systems by means of bipartite graphs. Using the DulmageMendelsohn decomposition, we are able to decompose our structured systems in such a way that in some special cases, the fixed controllable subspace can be obtained directly from the decomposition.
\end{abstract}

Keywords: Controllability, structured system theory, graph theory. 


\section{Introduction and motivation}

Our everyday life is now related with complex networks. Examples of such complex networks appear in biology, genetics, social networks, large communication or energy networks [25]. The recent interest of the network community for some concepts of control theory has raised a number of new control problems $[6,13,23]$. The controllability of networks has been studied in the framework of structured systems. This framework is well fitted for this type of study because it can take into account loosely defined large scale systems, and it is based on a graph representation of their structure. It is interesting to note that the problem of minimizing the action on a network to control it was converted in control theory into a very nice Minimal Controllability Problem which was solved very recently $[1,3,17,18,20]$. Besides these qualitative approaches, an energy minimisation paradigm has also been recently explored, see for example [28, 19]

This scientific context has renewed the interest for linear structured system theory and raised some interesting new problems in relation with controllability. Linear structured systems are dynamical systems for which the entries of the classical $(A, B)$ matrices are either zeroes or independent parameters. For such systems one can study generic (or structural) properties, i.e., properties which are true for almost any value of the nonzero parameters.

It happens that a lot of generic properties of structured systems can be related with a directed graph which is naturally associated with the structured system. In his fundamental paper [12], Lin laid the foundation of structured systems theory and gave a nice characterization of structural controllability in terms of particular graph objects, called cacti. The structural controllability conditions have been refined by several authors, see for example [22, 24], and are now well understood.

When the conditions for structural controllability are not satisfied, it is important to quantify to which extend the system is controllable. One way to do that is to consider the dimension of the controllable subspace. The generic dimension of the controllable subspace in graph-theoretic terms was given first in [10] and expanded in [21]. Notice that this dimension was used in network theory as a measure of the importance of a particular node

of driving the network, it is then called the control centrality of the node $[6,14,26]$. 
Our observation is that the dimension of the controllable subspace is certainly a question of interest but, since the controllable subspace is varying with the system parameters, the knowledge of this dimension does not say much about the possibility to reach a given state by a suitable control.

This is why we introduce the notion of the fixed controllable subspace ${ }^{1}$. This subspace contains all the fixed directions of the state space that can be reached and covered by a control, for almost any value of the system parameters. We provide a graphical characterization of this subspace which can be computed in polynomial time. We also propose a more efficient computation technique based on the Dulmage-Mendelsohn decomposition, but which (up to now) works only in particular cases. Incidently, the notion of fixed controllable subspace gives the possibility to define an associated fixed control centrality notion which may be of interest in network theory.

The outline of this paper is as follows. In Section 2 structured systems are recalled, together with some known results on structural controllability. Also some elementary observations on controllable subspaces are given, leading to the notion of fixed controllable subspace. In Section 3 a graph theory characterization of the fixed controllable subspace is derived. The results are illustrated by means of some examples in Section 4. The section also contains some remarks on the derived characterizations. In Section 5 an alternative characterization is proposed that possibly offers more insight in the way that the fixed controllable subspace can be constructed from unit vectors. Unfortunately, the proposed method can not (yet) be applied in all cases. The alternative characterization is illustrated in Section 6. In Section 7 the paper is concluded with some remarks and topics for future research.

\section{Structured systems and controllability aspects}

\subsection{Linear structured systems}

We consider a linear system with parameterized entries and denoted by $\Sigma_{\Lambda}$

$$
\Sigma_{\Lambda}: \dot{x}(t)=A_{\Lambda} x(t)+B_{\Lambda} u(t),
$$

where $x(t) \in \mathbb{R}^{n}$ is the state vector, $u(t) \in \mathbb{R}^{m}$ the input vector, and $A_{\Lambda}$ and $B_{\Lambda}$ are matrices of appropriate dimensions. The system is called a linear

\footnotetext{
${ }^{1}$ This is done for ease of terminology. It would have been more precise to call this subspace the fixed part of the controllable subspace.
} 
structured system if the entries of the composite matrix $J_{\Lambda}=\left[A_{\Lambda}, B_{\Lambda}\right]$ are either fixed zeros or independent parameters (not related by algebraic equations). The vector $\Lambda=\left(\lambda_{1}, \lambda_{2}, \ldots, \lambda_{k}\right)^{\top} \in \mathbb{R}^{k}$, with ${ }^{\top}$ indicating transposition, denotes the vector of independent (or free) parameters of the composite matrix $J_{\Lambda}$.

For linear structured systems one can study generic properties. A property is said to be generic (or structural) if it is true for all values of the parameter vector $\Lambda$ outside a proper algebraic variety in the parameter space $\mathbb{R}^{k}$, see [16]. This can then also be expressed by saying that the property is true for almost all values of the vector $\Lambda$, since a proper algebraic variety is a variety of zero measure.

For a structured matrix $M_{\Lambda}$, the rank of $M_{\Lambda}$ for almost any value of $\Lambda$, in the previous sense, is called its generic rank and denoted as $g$-rank $M_{\Lambda}$. Notice that $g$-rank $M_{\Lambda}$ is also the maximal value of rank $M_{\Lambda}$ for any value of $\Lambda$, for more details see [16].

A directed graph $G\left(\Sigma_{\Lambda}\right)=(Z, W)$ can be associated with the linear structured system $\Sigma_{\Lambda}(1)$ :

- the vertex set is $Z=X \cup U$, where $X$ and $U$ are the state and input sets given by $\left\{x_{1}, x_{2}, \ldots, x_{n}\right\}$ and $\left\{u_{1}, u_{2}, \ldots, u_{m}\right\}$, respectively,

- the edge set is $W=\left\{\left(x_{i}, x_{j}\right) \mid A_{\Lambda j i} \neq 0\right\} \cup\left\{\left(u_{i}, x_{j}\right) \mid B_{\Lambda j i} \neq 0\right\}$, where $A_{\Lambda j i}$ (resp. $\left.B_{\Lambda j i}\right)$ denotes the entry $(j, i)$ of the matrix $A_{\Lambda}\left(\operatorname{resp} . B_{\Lambda}\right)$.

Recall that a path in $G\left(\Sigma_{\Lambda}\right)$ from a vertex $z_{i_{0}}$ to a vertex $z_{i_{q}}$ is a sequence of edges $\left(z_{i_{0}}, z_{i_{1}}\right),\left(z_{i_{1}}, z_{i_{2}}\right), \ldots,\left(z_{i_{q-1}}, z_{i_{q}}\right)$, such that $z_{i_{t}} \in Z$ for $t=0,1, \ldots, q$ and $\left(z_{i_{t-1}}, z_{i_{t}}\right) \in W$ for $t=1,2, \ldots, q$. If $z_{i_{0}} \in U$ and, $z_{i_{q}} \in X$, the path is called an input-state path. A path for which $z_{i_{0}}=z_{i_{q}}$ is called a circuit. A stem is an input-state path which does not meet the same vertex twice. A system is said to be input-connected if any state vertex is the end vertex of a stem. A cycle is a circuit which does not meet the same vertex twice, except for the initial/end vertex. Two paths are disjoint when they cover disjoint set of vertices. When some stems and cycles are mutually disjoint, they constitute a set of disjoint stems and cycles.

Example 1. Consider the structured system $\Sigma_{\Lambda}$ with four states and one 
input, whose parameterized matrices $A_{\Lambda}$ and $B_{\Lambda}$ are defined as follows.

$$
A_{\Lambda}=\left(\begin{array}{cccc}
\lambda_{1} & 0 & 0 & 0 \\
0 & 0 & 0 & 0 \\
\lambda_{2} & 0 & 0 & 0 \\
0 & \lambda_{3} & 0 & \lambda_{4}
\end{array}\right), \quad B_{\Lambda}=\left(\begin{array}{c}
\lambda_{5} \\
\lambda_{6} \\
0 \\
0
\end{array}\right)
$$

The corresponding graph is given in Figure 1. The path $\left(u_{1}, x_{1}, x_{3}\right)$ with the

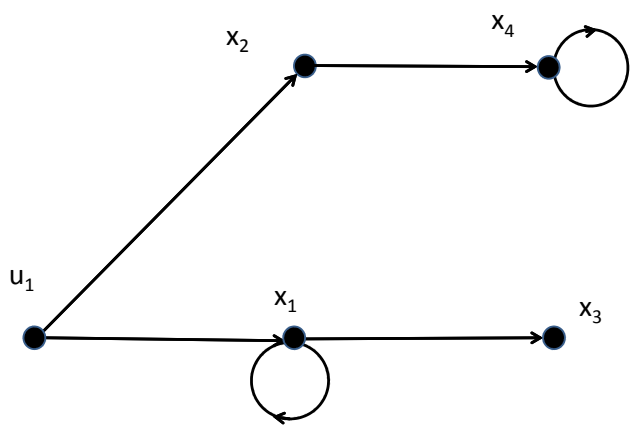

Figure 1: Graph $G\left(\Sigma_{\Lambda}\right)$ of Example 1

circuit on $x_{4}$ constitute a set of disjoint stems and cycles for $G\left(\Sigma_{\Lambda}\right)$.

The notion of structural controllability was introduced and studied by Lin who proved a necessary and sufficient condition for structural controllability in terms of graph-theoretic objects called cacti, see [12]. The following result can proved to be equivalent to Lin's result, see for instance [24, 22].

Theorem 1. Let $\Sigma_{\Lambda}$ be the linear structured system defined by (1) with associated graph $G\left(\Sigma_{\Lambda}\right)$. The system is structurally controllable if and only if

- the system $\Sigma_{\Lambda}$ is input-connected,

- $g$-rank $\left[A_{\Lambda}, B_{\Lambda}\right]=n$.

In Example 1, the graph of $\Sigma_{\Lambda}$ is clearly input connected and the first controllability condition is satisfied. The second condition is a little less obvious, 
but it can be noticed that in

$$
\left[A_{\Lambda}, B_{\Lambda}\right]=\left(\begin{array}{ccccc}
\lambda_{1} & 0 & 0 & 0 & \lambda_{5} \\
0 & 0 & 0 & 0 & \lambda_{6} \\
\lambda_{2} & 0 & 0 & 0 & 0 \\
0 & \lambda_{3} & 0 & \lambda_{4} & 0
\end{array}\right)
$$

column 3 being null and columns 2 and 4 being dependent, the generic rank of $\left[A_{\Lambda}, B_{\Lambda}\right]$ cannot be more than 3. Taking, for example, $\lambda_{1}=\lambda_{2}=\lambda_{3}=$ $\lambda_{4}=\lambda_{5}=\lambda_{6}=1$ gives a rank 3 for $\left[A_{\Lambda}, B_{\Lambda}\right]$, therefore the generic rank of $\left[A_{\Lambda}, B_{\Lambda}\right]$ is 3 and the system is not controllable. In Section 5 , we will see a graph characterization of this rank condition.

\subsection{Controllable subspace}

When the conditions of Theorem 1 are not satisfied, it is of interest to characterize the controllable subspace of the system (1). This controllable subspace is defined as

$$
\mathcal{C}_{\Lambda}=\operatorname{Im}\left[B_{\Lambda}, A_{\Lambda} B_{\Lambda}, \ldots, A_{\Lambda}^{n-1} B_{\Lambda}\right]
$$

The problem was studied first in [10], where the dimension of the controllable subspace was given both in algebraic and graphical terms. We just recall here the graph-theoretic result [10]

Theorem 2. Let $\Sigma_{\Lambda}$ be the input-connected linear structured system defined by (1) with associated graph $G\left(\Sigma_{\Lambda}\right)$. The generic dimension of the controllable subspace (4) is given by the maximal number of state vertices which can be covered by a disjoint set of stems and cycles in $G\left(\Sigma_{\Lambda}\right)$.

This nice graph theoretic characterization was proved to be checkable in polynomial time in [21]. For more on complexity issues, see [8].

However, even if the dimension of the controllable subspace is constant for almost any value of the parameters in $\Lambda$, the subspace itself will vary with these parameters. The knowledge of the dimension of $\mathcal{C}_{\Lambda}$ does not say much about the possibility to reach generically a given state by a suitable control. We will then try to characterize the set of given states which can be reached generically in the system $\Sigma_{\Lambda}$. This set has obviously a linear subspace structure. We will call it the fixed controllable subspace of $\Sigma_{\Lambda}$ and denote it as $\mathcal{C}_{F}$. It is clear that a vector in $\mathcal{C}_{F}$ must be reachable for almost

any value of $\Lambda$, therefore $\mathcal{C}_{F}$ is the intersection of the controllable subspaces 
$\mathcal{C}_{\Lambda}$ for almost all values of $\Lambda$. A similar notion was introduced in [4] for the study of the disturbance decoupling problem.

If we denote by $X_{I C}$ the subspace generated by the unit vectors corresponding to the input-connected vertices, we obviously have that:

$$
\mathcal{C}_{F} \subset \mathcal{C}_{\Lambda} \subset X_{I C} \subset X
$$

The unit vectors corresponding to the non input-connected vertices are obviously not controllable. Thus, our object of interest remains in $X_{I C}$. Therefore, we will assume, without loss of generality, in the following that the system $\Sigma_{\Lambda}$ is input-connected, i.e., that $X_{I C}=X$.

\section{The fixed controllable subspace}

In this section, we will give two technical lemmas and a Theorem which provides with a complete characterization of the fixed controllable subspace.

If $\bar{x}$ is some fixed vector, we denote by $\bar{B}_{\Lambda \bar{x}}$ the input matrix defined as $\bar{B}_{\Lambda \bar{x}}=\left[B_{\Lambda}, \bar{x}\right]$. We also introduce the controllable subspace

$$
\mathcal{C}_{\Lambda \bar{x}}=\operatorname{im}\left[\bar{B}_{\Lambda \bar{x}}, A_{\Lambda} \bar{B}_{\Lambda \bar{x}}, \ldots, A_{\Lambda}^{n-1} \bar{B}_{\Lambda \bar{x}}\right] .
$$

Lemma 1. Consider the linear structured system defined by (1). Let $\bar{x} \in X$ be a fixed vector. Then $\bar{x} \in \mathcal{C}_{F}$ if and only if, for almost any $\Lambda$,

$$
\mathcal{C}_{\Lambda \bar{x}}=\mathcal{C}_{\Lambda}
$$

Proof:

$\Rightarrow$ First, it is clear that $\mathcal{C}_{\Lambda} \subset \mathcal{C}_{\Lambda \bar{x}}$. The controllable subspace $\mathcal{C}_{\Lambda}$ being an $A_{\Lambda}$-invariant, it follows that if $\bar{x} \in \mathcal{C}_{F} \subset \mathcal{C}_{\Lambda}$, we also have $A_{\Lambda}^{j} \bar{x} \in \mathcal{C}_{\Lambda}$ for $j=0, \ldots, n-1$, implying that $\mathcal{C}_{\Lambda \bar{x}} \subset \mathcal{C}_{\Lambda}$. Therefore, adding this new input will not increase the dimension of the controllable subspace $\mathcal{C}_{\Lambda}$ and the result follows.

$\Leftarrow$ Let $\bar{x} \in X$ be fixed and assume that $\mathcal{C}_{\Lambda}=\mathcal{C}_{\Lambda \bar{x}}$ for almost all $\Lambda$. By (6) it follows that $\bar{x} \in \mathcal{C}_{\Lambda \bar{x}}=\mathcal{C}_{\Lambda}$ for almost all $\Lambda$. Hence, $\bar{x} \in \mathcal{C}_{F}$.

A similar result as Lemma 1 was developed in [27], in the context of state transittability.

Lemma 2. Consider the linear structured system defined by (1). Then $\bar{x} \in$ $\mathcal{C}_{F}$ if and only if the unit vector $v_{i} \in \mathcal{C}_{F}$ for any $i$ such that the $i$-th entry $\bar{x}_{i}$ of $\bar{x}$ is nonzero. 
Proof:

$\Leftarrow$ If the unit vector $v_{i} \in \mathcal{C}_{F}$ for any $i$ such that the $i$-th entry $\bar{x}_{i}$ of $\bar{x}$ is nonzero, then $\bar{x}$, which is linear combination of these unit vectors, also belongs to $\mathcal{C}_{F}$ from linearity arguments.

$\Rightarrow$ Define a new structured system $\bar{\Sigma}_{\bar{\Lambda}}$ with associated graph $G\left(\bar{\Sigma}_{\bar{\Lambda}}\right)$ by adding a new input vertex $\bar{u}$ with an edge $\left(\bar{u}, x_{i}\right)$ for all vertices $x_{i}$ such that the $i$-th entry $\bar{x}_{i}$ of $\bar{x}$ is nonzero. This defines a new column $\bar{b}$ in the $B$ matrix which has the same zero/nonzero structure as $\bar{x}$.

Suppose now that for some $i$ such that $\bar{x}_{i} \neq 0$, we have $v_{i} \notin \mathcal{C}_{F}$. From Lemma 1 it follows that the controllable subspace of the pair $\left(A_{\Lambda},\left[B_{\Lambda}, v_{i}\right]\right)$ is larger than $\mathcal{C}_{\Lambda}$. The graph of the pair $\left(A_{\Lambda},\left[B_{\Lambda}, v_{i}\right]\right)$ is obtained from $G\left(\Sigma_{\Lambda}\right)$ by adding a new input vertex $\bar{u}$ and an edge $\left(\bar{u}, x_{i}\right)$. From Theorem 2 , this graph contains a set of stems and cycles which covers at least $\mu+1$ state vertices if $\mu$ is the generic dimension of $\mathcal{C}_{\Lambda}$. From this, it follows that there is also a set of stems and cycles which covers at least $\mu+1$ state vertices in the graph $G\left(\bar{\Sigma}_{\bar{\Lambda}}\right)$. Therefore, the generic dimension of the controllable subspace of $\bar{\Sigma}_{\bar{\Lambda}}$ is at least $\mu+1$. With $\bar{b}$ and $\bar{x}$ having the same structure, it follows from Lemma 1 that $\bar{x}$ is almost surely not in $\mathcal{C}_{F}$, since adding $\bar{x}$ gives an extra dimension. So, for all $\bar{x}_{i} \neq 0$, we have that $v_{i} \in \mathcal{C}_{F}$.

From Lemmas 1 and 2, we can then deduce a complete characterization of the fixed controllable subspace as follows.

Theorem 3. Let $\Sigma_{\Lambda}$ be the input-connected linear structured system, defined by (1) with associated graph $G\left(\Sigma_{\Lambda}\right)$. The fixed controllable subspace $\mathcal{C}_{F}$ is generated by unit vectors in the state space defined as follows:

The unit vector $v_{i} \in \mathcal{C}_{F}$ if and only if adding a new input $\bar{u}$ and an edge $\left(\bar{u}, x_{i}\right)$ to $G\left(\Sigma_{\Lambda}\right)$ does not increase the maximal number of state vertices which can be covered by a disjoint set of stems and cycles in the graph.

Remark 1. In simple terms, Theorem 3 says the following.

- The fixed controllable subspace $\mathcal{C}_{F}$ is in one to one correspondence with a set of vertices in $G\left(\Sigma_{\Lambda}\right)$, which is not the case for $\mathcal{C}_{\Lambda}$.

- A vertex is in the fixed controllable subspace if and only if, adding an input on this vertex does not change the controllable subspace, which can be checked by Theorem 2. 
- Since the dimension of the controllable subspace can be computed in a polynomial way, the fixed controllable subspace can also be obtained in a polynomial time.

\section{Examples and remarks}

With Remark 1 in mind, Theorem 3 is illustrated on Example 1.

Example 1 (cont.) From Theorem 2, it can be seen that the controllable subspace of this system has generic dimension 3. Indeed, the stem $\left(u_{1}, x_{1}, x_{3}\right)$ and the circuit on $x_{4}$ cover 3 states, while it is not possible to cover all the states by a disjoint set of stems and cycles. Other ways to cover 3 state vertices are either to use the stem $\left(u_{1}, x_{2}, x_{4}\right)$ and the cycle on $x_{1}$, or to use the stem $\left(u_{1}, x_{2}\right)$ and the cycles on $x_{1}$ and $x_{4}$. There are no other possibilities.

To test if $v_{2}$ belongs to the fixed controllable subspace, we add a virtual input $\bar{u}_{2}$ and an edge $\left(\bar{u}_{2}, x_{2}\right)$. Now the four state vertices can be covered by the stems $\left(u_{1}, x_{1}, x_{3}\right)$ and $\left(\bar{u}_{2}, x_{2}, x_{4}\right)$, so that the controllable subspace has now dimension 4. It follows from Lemma 2 that $v_{2}$ does not belong to the fixed controllable subspace. We get the same conclusion for $v_{1}$ and $v_{3}$. It can be seen that adding an input to vertex $x_{4}$ will not increase the dimension of the controllable subspace. Therefore $\mathcal{C}_{F}=\operatorname{span}\left\{v_{4}\right\}$.

It could be tempting to conjecture that vertices which correspond to the fixed controllable subspace are those which belong to all the possible coverings of size 3. This is not the case, since in our example $x_{1}$ and $x_{4}$ belong to the three possible coverings but $x_{1}$ does not correspond to the fixed controllable subspace span $\left\{v_{4}\right\}$. Nevertheless, the converse result is true: the vertices which do not belong to all the possible coverings of maximal size cannot correspond to directions in the fixed controllable subspace. Indeed, if a vertex $x_{i}$ does belong to a possible covering of maximal size, then adding a virtual input $\bar{u}_{i}$ and an edge $\left(\bar{u}_{i}, x_{i}\right)$ leads to cover a greater number of state vertices using disjoint stems and cycles. Therefore adding an input to vertex $x_{i}$ will increase the dimension of the controllable subspace and so the corresponding unit vector $v_{i}$ does not belong to the fixed controllable subspace.

Remark 2. In the literature, a linear structured system is called strongly structural controllable if it is controllable for every nonzero value of its free parameters, see [15]. Hence, in contrast to ordinary structural controllabil-

ity all parameters $\lambda_{i}, i=1, \ldots, k$, must be taken into account with nonzero 
values. So, a set of zero measure of parameter value combinations for which the system is not controllable is not allowed. The notion of strong structural controllability is another way to get rid of the assumption of independence of the parameters in structured systems. However, the fixed controllable subspace of the systems in the context of this paper has nothing to do with strong structural controllability and the strong structurally controllable subspace, see [11, 26].

Remark 3. In [21] it is proved that the generic dimension of the controllable subspace can be computed by means of polynomial techniques, and more specifically linear programming methods. As indicated above the elements in the fixed controllable part can be determined by means of computing this dimension for extended systems. Indeed, according to Lemma 1, the $i$-th unit vector is contained in the fixed part if and only if the generic dimension of the system with extended input matrix $\left[B_{\Lambda}, v_{i}\right]$ is the same as the generic dimension of the controllable subspace of the original system. Hence, checking this fact requires two times the application of a linear program. So, the results in this paper can be verified by means of computationally efficient methods coming from optimization and network theory.

\section{Another characterization of the fixed controllable subspace}

In the previous section, we gave a polynomial way to compute the fixed controllable subspace. However, to check if a state vertex belongs to this fixed subspace, we have to use Hosoe's condition which needs solving a maximum matching problem with maximum cost. In this section we provide with a less costly method.

We recall first the graph characterization of $g$-rank $\left[A_{\Lambda}, B_{\Lambda}\right]$. This generic rank is computed using the bipartite graph associated with the system. This bipartite graph, together with its so-called Dulmage-Mendelsohn decomposition (DM-decomposition), introduced in [7], will give information on the fixed controllable subspace of $\Sigma_{\Lambda}$ and, in an important particular case, a complete characterization of the fixed subspace.

\subsection{Bipartite graph and DM-decomposition}

The bipartite graph $V\left(\Sigma_{\Lambda}\right)=\left(V^{+}, V^{-} ; W^{\prime}\right)$ associated with $\Sigma_{\Lambda}$ is defined as follows. The vertex set $V^{+}$is given by $X^{+} \cup U$, the vertex set $V^{-}$is given by $X^{-}$, with $X^{+}=\left\{x_{1}^{+}, \ldots, x_{n}^{+}\right\}$the first set of state vertices, $X^{-}=$ 
$\left\{x_{1}^{-}, \ldots, x_{n}^{-}\right\}$the second set of state vertices and $U=\left\{u_{1}, \ldots, u_{m}\right\}$ the set of input vertices. Notice that here we have split each state vertex $x_{i}$ of $G\left(\Sigma_{\Lambda}\right)$ into two vertices $x_{i}^{+}$and $x_{i}^{-}$. The edge set $W^{\prime}$ is described by $W_{A} \cup W_{B}$ with $W_{A}=\left\{\left(x_{j}^{+}, x_{i}^{-}\right) \mid A_{\Lambda i j} \neq 0\right\}$ and $W_{B}=\left\{\left(u_{j}, x_{i}^{-}\right) \mid B_{\Lambda i j} \neq 0\right\}$. In the former, for instance $A_{\Lambda i j} \neq 0$ means that the $(i, j)$-th entry of the matrix $A_{\Lambda}$ is a parameter (structurally nonzero).

A matching in a bipartite graph $V\left(\Sigma_{\Lambda}\right)=\left(V^{+}, V^{-} ; W^{\prime}\right)$ is an edge set $M \subseteq W^{\prime}$ such that the edges in $M$ have no common vertex. The cardinality of a matching, i.e., the number of edges it consists of, is also called its size. A matching $M$ is called maximum if its cardinality is maximum. In general, a maximum matching is not unique. The maximum matching problem is the problem of finding such a matching of maximal cardinality. The following result can be found in [5].

Proposition 1. Let $\Sigma_{\Lambda}$ be the linear structured system defined by (1) with associated bipartite graph $V\left(\Sigma_{\Lambda}\right)$. The generic rank of $\left[A_{\Lambda}, B_{\Lambda}\right]$ is equal to the size of a maximal matching in $V\left(\Sigma_{\Lambda}\right)$. In particular, the generic rank of $\left[A_{\Lambda}, B_{\Lambda}\right]=n$ if and only if there exists a size $n$ matching in $V\left(\Sigma_{\Lambda}\right)$.

Example 1 (cont.) The bipartite graph $V(\Sigma)$ of Example 1 is given in Figure 2. A maximal matching consists of three edges. Indeed, $\left\{\left(u_{1}, x_{1}^{-}\right)\right.$,

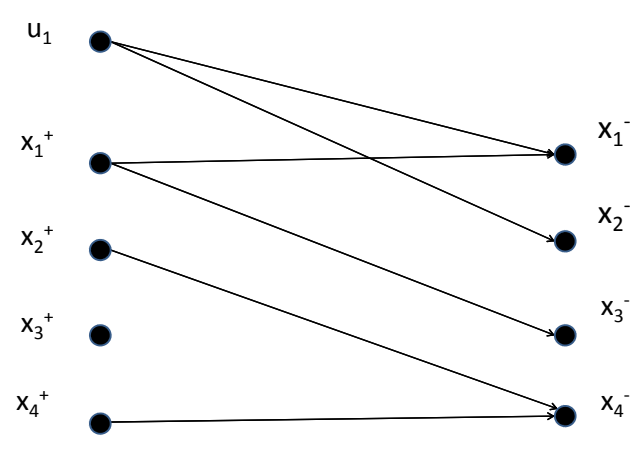

Figure 2: Bipartite graph of Example 1

$\left.\left(x_{1}^{+}, x_{3}^{-}\right),\left(x_{4}^{+}, x_{4}^{-}\right)\right\}$is a set of three disjoint edges, and any set of four edges has (at least) two edges that have a common node. Therefore, it follows by Proposition 1 that the $g$-rank $\left[A_{\Lambda}, B_{\Lambda}\right]=3$. 
A useful tool to parameterize all the maximum matchings in a bipartite graph is the DM-decomposition which will be presented now. The DM-decomposition allows to decompose a (general) bipartite graph $V=$ $\left(V^{+}, V^{-} ; W^{\prime}\right)$ into a uniquely defined family of bipartite subgraphs $V_{i}=$ $\left(V_{i}^{+}, V_{i}^{-} ; W_{i}^{\prime}\right), i=0,1, \ldots, r, \infty$, called the DM-components, where $\left(V_{0}^{+}, V_{1}^{+}\right.$, $\left.\cdots, V_{r}^{+}, V_{\infty}^{+}\right)$is a partition of $V^{+}$, and likewise for $V^{-}$and $W^{\prime}$. The component $V_{0}$ is called minimal inconsistent part, the component $V_{\infty}$ is called maximal inconsistent part and the remaining components are called consistent parts. The names minimal and maximal inconsistent part can be motivated by the ordering that can be put on the components. Indeed, it is possible to define an ordering $\prec$ such that $V_{0} \prec V_{i} \prec V_{\infty}$ for all $i=1, \ldots, r$, see [16] for more details. In the latter reference also the following properties of the DM-components can be found.

Proposition 2. Let $V=\left(V^{+}, V^{-} ; W^{\prime}\right)$ be a bipartite graph and its $D M$ decomposition with $V_{i}=\left(V_{i}^{+}, V_{i}^{-} ; W_{i}^{\prime}\right), i=0,1, \ldots, r, \infty$, as its DM-components. Then we have the following properties:

1. A maximum matching on $V$ is a union of maximum matchings on the DM-components $V_{i}, i=0,1, \ldots, r, \infty$.

2. A vertex $v$ in $V_{0}^{-}$(in $V_{\infty}^{+}$, in $V_{i}^{+}$or $\left.V_{i}^{-}, i=1, \ldots, r\right)$ is covered by any maximum matching on $V$.

3. A vertex $v$ in $V^{+}$belongs to the minimal inconsistent part $V_{0}^{+}$if and only if there exists a maximum matching on $V$ that does not cover $v$.

4. A vertex $v$ in $V^{-}$belongs to the maximal inconsistent part $V_{\infty}^{-}$if and only if there exists a maximum matching on $V$ that does not cover $v$.

Here we are interested in the effect of adding a new input $\bar{u}$ to the system $\Sigma_{\Lambda}$, this new input acting only on the state vertex $x_{i}$. Denote by $\bar{B}_{\Lambda}^{i}$ the associated new input matrix. From the properties of the DM-decomposition, the following result can be obtained, see [2] for more details.

Lemma 3. The equation g-rank $\left[A_{\Lambda}, \bar{B}_{\Lambda}^{i}\right]=g$-rank $\left[A_{\Lambda}, B_{\Lambda}\right]+1$ holds if and only if $x_{i}^{-}$belongs to the $V_{\infty}^{-}$set of the DM-decomposition of $V\left(\Sigma_{\Lambda}\right)$. Otherwise, $g$-rank $\left[A_{\Lambda}, \bar{B}_{\Lambda}^{i}\right]=g$-rank $\left[A_{\Lambda}, B_{\Lambda}\right]$.

Finding the DM-decomposition of a bipartite graph requires first obtaining a maximum matching in the graph and, in a second step, using alternating path techniques. Since the alternating path techniques are of lower complexity, the complexity of obtaining the DM-decomposition is just as the 
complexity of maximum matching algorithm [9]. Clearly, this is of lower complexity than computing a maximum matching with maximum weight as is required in Hosoe's algorithm.

\subsection{Nodes which are not in the fixed controllable subspace}

From the previous subsection, we know that the rank of the matrix $\left[A_{\Lambda}, B_{\Lambda}\right]$ can be computed by using the corresponding bipartite graph $V\left(\Sigma_{\Lambda}\right)$ and its DM-decomposition. This allows to characterize nodes which do not belong to the fixed controllable subspace.

Proposition 3. Let $\Sigma_{\Lambda}$ be the linear structured system defined by (1) with associated bipartite graph $V\left(\Sigma_{\Lambda}\right)$ and its DM-decomposition. If $x_{i}^{-}$belongs to the $V_{\infty}^{-}$set of the DM-decomposition of $V\left(\Sigma_{\Lambda}\right)$, then $x_{i}$ does not belong to the fixed controllable subspace.

Proof:

Let $x_{i}$ be such that $x_{i}^{-}$belongs to the $V_{\infty}^{-}$set of the DM-decomposition of $V\left(\Sigma_{\Lambda}\right)$. From Lemma 3, it follows that adding an input on this vertex will increase the generic rank of $\left[A_{\Lambda}, B_{\Lambda}\right]$. Therefore $g$-rank $\left[A_{\Lambda}, B_{\Lambda}, v_{i}\right]=$ $g$-rank $\left[A_{\Lambda}, B_{\Lambda}\right]+1$, where $v_{i}$ is the corresponding unit vector, and we have that $v_{i} \notin \operatorname{im}\left[A_{\Lambda}, B_{\Lambda}\right]$. Since $\mathcal{C}_{\Lambda} \subset \operatorname{im}\left[A_{\Lambda}, B_{\Lambda}\right]$, vector $x_{i}$ does not belong to the fixed controllable subspace.

\subsection{When $\operatorname{dim}\left(\mathcal{C}_{\Lambda}\right)=g-\operatorname{rank}\left[A_{\Lambda}, B_{\Lambda}\right]$}

In this subsection, we characterize the fixed controllable subspace in the particular case that $\operatorname{dim}\left(\mathcal{C}_{\Lambda}\right)=\mathrm{g}-\operatorname{rank}\left[A_{\Lambda}, B_{\Lambda}\right]$.

Proposition 4. Let $\Sigma_{\Lambda}$ be the linear structured system defined by (1) with associated bipartite graph $V\left(\Sigma_{\Lambda}\right)$ and its DM-decomposition. Assume that $\operatorname{dim}\left(\mathcal{C}_{\Lambda}\right)=g$-rank $\left[A_{\Lambda}, B_{\Lambda}\right]$. Then $x_{i}$ belongs to the fixed controllable subspace if and only if $x_{i}^{-}$does not belong to the $V_{\infty}^{-}$set of the DM-decomposition of $V\left(\Sigma_{\Lambda}\right)$.

Proof:

In this case, it follows from Lemma 3 that adding an input on this vertex will not increase the generic rank of $\left[A_{\Lambda}, B_{\Lambda}\right]$. Then for the corresponding unit vector $v_{i}$, we have $g$-rank $\left[A_{\Lambda}, B_{\Lambda}, v_{i}\right]=g$-rank $\left[A_{\Lambda}, B_{\Lambda}\right]$ and $v_{i} \in \operatorname{im}\left[A_{\Lambda}, B_{\Lambda}\right]=\mathcal{C}_{\Lambda}$. Therefore, $x_{i}$ belongs to the fixed controllable subspace. Conversely, the necessity of the condition follows from Proposition 3. $\triangle$. 
This means that under the condition $\operatorname{dim}\left(\mathcal{C}_{\Lambda}\right)=g$-rank $\left[A_{\Lambda}, B_{\Lambda}\right]$, the DM-decomposition provides with a complete characterization of the fixed controllable subspace.

\subsection{When $\operatorname{dim}\left(\mathcal{C}_{\Lambda}\right)<g-\operatorname{rank}\left[A_{\Lambda}, B_{\Lambda}\right]$}

For this case, we cannot conclude in general and, in particular, there may exist nodes $x_{i}$ such that $x_{i}^{-}$does not belong to the $V_{\infty}^{-}$set of the DMdecomposition of $V\left(\Sigma_{\Lambda}\right)$ and which are not in the the fixed controllable subspace. This can be seen from the second example in the next section.

\section{Further examples}

In this section we illustrate the characterization based on the DM-decomposition of the bipartite system graph. We begin by reconsidering Example 1.

Example 1 (cont.) Consider the bipartite graph $V(\Sigma)$ of Example 1 and a DM-decomposition given in Figure 3. Before we showed that by using Propo-

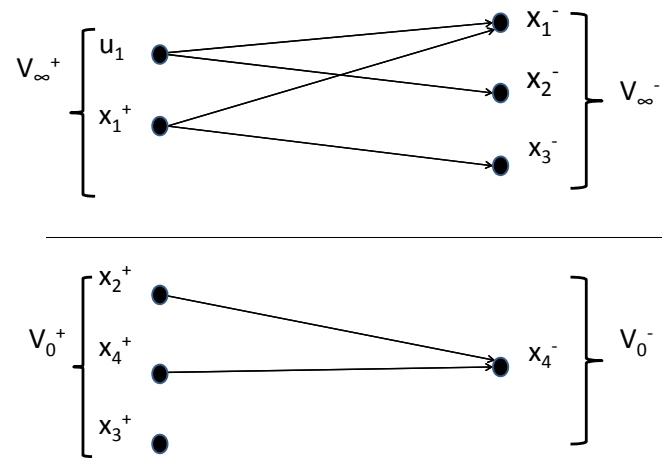

Figure 3: DM-decomposition for Example 1

sition 1 it follows that the $g-\operatorname{rank}\left[A_{\Lambda}, B_{\Lambda}\right]=3$. From Example 1 we know that the generic dimension of the controllable subspace is also 3. Hence, we are in position to apply Proposition 4. It follows from this proposition and Figure 3 that the only node that belongs to the fixed controllable subspace is $x_{4}$. This coincides with the result of Theorem 3. There the conclusion was obtained by (laborously) looking for the effect of additional virtual inputs. Here it follows directly from the DM-decomposition. 
Unfortunately, the characterization in the previous section is not (yet) complete, as can be seen by means of the following example.

Example 2. Consider the example whose graph and bipartite graph are depicted in Figure 4. This system has one input, four states and is inputconnected, as can be seen from the graph.

From Hosoe's Theorem applied to the graph, it follows that the controllable subspace has dimension 2. Indeed, the stems $\left(u_{1}, x_{1}, x_{2}\right)$ or $\left(u_{1}, x_{3}, x_{4}\right)$ can be used, i.e., $\operatorname{dim}\left(\mathcal{C}_{\Lambda}\right)=2$. From the bipartite graph $V\left(\Sigma_{\Lambda}\right)$ and its $D M$ decomposition, it follows that $g$-rank $\left[A_{\Lambda}, B_{\Lambda}\right]=3$.

Hence, $\operatorname{dim}\left(\mathcal{C}_{\Lambda}\right)<g$-rank $\left[A_{\Lambda}, B_{\Lambda}\right]$. Moreover, $x_{1}$ and $x_{3}$, which belong to the $V_{\infty}^{-}$set of the DM-decomposition, are not in the fixed controllable subspace, as follows from Proposition 3. The result of Proposition 4 cannot be used. Indeed, it can be easily checked in the graph that adding an input on $x_{2}$ or $x_{4}$ increases the dimension of the controllable subspace with one. Therefore, also $x_{2}$ and $x_{4}$ are not in the fixed controllable subspace.

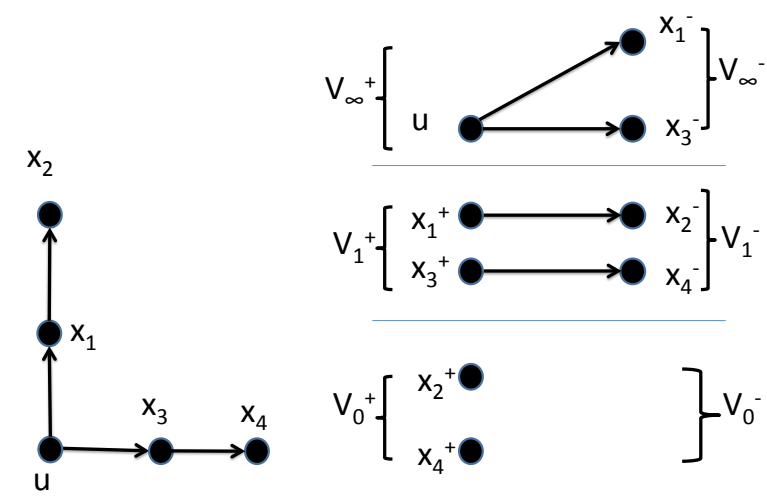

Figure 4: Graph (left) and bipartite graph of Example 3 with DM-decomposition (right)

\section{Conclusions}

In this paper we have introduced the notion of fixed controllable subspace of a structured system. We have represented the structured systems by means of graphs. The controllable subspace of a structured system in general contains a fixed part and a part that varies with the values of the free parameters that represent the nonzero entries in the system matrices. The 
fixed controllable subspace stands for the subspace spanned by the collection of unit vectors in state space that for almost all values of the free parameters are contained in the controllable subspace.

We have characterized how the fixed part can be determined using the well-known result of Hosoe on the generic dimension of the controllable subspace. It turns out that a unit state vector is element of the fixed part if and only if the dimension of the controllable subspace does not increase in case the corresponding node is considered to be an additional driver node, i.e., a node that is controlled by means of an additional control.

Since the result of Hosoe can be computed by means of a linear programming methods, it follows that by similar techniques it can be checked which unit state vectors belong to the fixed part of the controllable subspace. Hence, the fixed controllable subspace can be computed by means of efficient and well-known techniques for optimization and network techniques.

Clearly, the above approach is computationally oriented. It turns out that also more insightful methods can be applied. These methods involve the use of the so-called DM-decomposition. However, up to now these methods could only be worked out for some special cases. The characterization for the general case remains a topic for further research. Also the notion of fixed control centrality will be a topic for future investigations.

\section{References}

[1] S. Assadi, S. Khanna, Y. Li, and V. M. Preciado. Complexity of the minimum input selection problem for structural controllability. In 5th IFAC Workshop on Distributed Estimation and Control in Networked Systems, NecSys, Philadelphia, 2015.

[2] C. Commault and J. M. Dion. Input addition and leader selection for the controllability of graph-based systems. Automatica, 49(11):3322-3328, 2013.

[3] C. Commault and J. M. Dion. The single-input minimal controllability problem for structured systems. Syst. Cont. Lett., 80:50-55, 2015.

[4] C. Commault, J. M. Dion, and V. Hovelaque. A geometric approach for structured systems: application to the disturbance decoupling problem. Automatica, 33:403-409, 1997. 
[5] C. Commault, J. M. Dion, and J. van der Woude. Characterization of generic properties of linear structured systems for efficient computations. Kybernetika, 38(5):503-520, 2002.

[6] J. Ding and Y-Z Lu. Control backbone: An index for quantifying a node's importance for the network controllability. Neurocomputing, 153:309-318, 2015.

[7] A. L. Dulmage and N. S. Mendelsohn. Coverings of bipartite graphs. Canad. J. Math, 10:517-534, 1958.

[8] M.R. Garey and D.S. Johnson. Computers and intractability: A guide to the theory of NP-Completeness. W.H. Freeman And Company, 1999.

[9] J.E. Hopcroft and R.M. Karp. An $n^{5 / 2}$ algorithm for maximum matchings in bipartite graphs. SIAM J. on Comp., 2:225-231, 1973.

[10] S. Hosoe. Determination of generic dimensions of controllable subspaces and its application. IEEE Trans. Automat. Control, AC-25:1192-1196, 1980 .

[11] D.C. Jarczyk, F. Svaricek, and B. Alt. Determination of the dimensions of strong structural controllability subspaces. In 4th IFAC Symposium Systems Structure and Control, Ancona, 2010.

[12] C. T. Lin. Structural controllability. IEEE Trans. Automat. Control, 19(3):201-208, 1974.

[13] Y. Y. Liu, J. J. Slotine, and A. L. Barabasi. Controllability of complex networks. Nature, 473:167-173, 2011.

[14] Y. Y. Liu, J. J. Slotine, and A. L. Barabasi. Control centrality and hierarchical structure in complex networks. PLOS ONE, 7(9), 2012.

[15] H. Mayeda and T. Yamada. Strong structural controllability. SIAM J. Cont. Optim., 17(1):123-138, 1979.

[16] K. Murota. Systems Analysis by Graphs and Matroids, volume 3 of Algorithms and Combinatorics. Springer-Verlag New-York, Inc., 1987.

[17] A. Olshevsky. Minimal controllability problems. IEEE Trans. on Control of Network Systems, 1(3):249-258, 2014. 
[18] A. Olshevsky. Minimum input selection for structural controllability. In American Control Conference (ACC), pages 2218-2223, 2015.

[19] F. Pasqualetti, S. Zampieri, and F. Bullo. Controllability metrics, limitations and algorithms for complex networks. In Proceedings of the American Control Conference (ACC), pages 3287-3292, Portland, 2014.

[20] S. Pequito, S. Kar, and P. Aguiar. A framework for structural input/output and control configuration selection in large-scale systems. IEEE Trans. Automat. Control, 61(2):303-318, 2016.

[21] S. Poljak. On the generic dimension of controllable subspaces. IEEE Trans. Automat. Control, 35(3):367-369, 1990.

[22] K.J. Reinschke. Multivariable control: a graph-theoretic approach. Springer Verlag, 1988.

[23] J. Ruths and D. Ruths. Control profiles of complex networks. Science, 343:1373-1375, 2014.

[24] R.W. Shields and J.B. Pearson. Structural controllability of multi-input linear systems. IEEE Trans. Automat. Control, AC-21:203-212, 1976.

[25] S. Strogatz. Exploring complex networks. Nature, 410:268-276, 2001.

[26] C. Tu. Strong structural centrality of a complex network. Physica Scripta, 90(3), 2015.

[27] F.X. Wu, J. Wang, J. Liu, and L. Chen. Transittability of complex networks and its applications to regulatory biomolecular networks. Scientifc Reports, April(4819), 2014.

[28] G. Yan, J. Ren, Y-C. Lai, C-H. Lai, and B. Li. Controlling complex networks: how much energy is needed ? Phys. Rev. Lett., 108(21):218703, 2012 . 